\title{
Compact, Field-Portable Smartphone Chiral Molecule Concentration Estimation System via Multi-sensor Computational Polarimetry
}

Shahid A. Haider

Farnoud Kazemzadeh

Alexander Wong
University of Waterloo, ON, Canada

University of Waterloo, ON, Canada

University of Waterloo, ON, Canada

\begin{abstract}
In this paper, we present a compact, field-portable smartphone chiral molecule concentration estimation system based on the principle of multi-sensor computational polarimetry. The presented system was designed as an attachment for a smartphone, thus leveraging the computational power to achieve full autonomy and small form factor, while greatly reducing the cost of the system. In addition, by leveraging Maul's Law, the size and complexity of the presented system can be greatly reduced, consisting of just four static components: i) a diode laser source, ii) a linear polariser, iii) a cell for chiral solution, and iv) a linear analyser. Finally, the high megapixel count of the smartphone camera is leveraged via multisensor computational polarimetry, where a multitude of measurements by different sensors are made in a single acquisition to enhance the estimation of the angle of linear polarisation, and thereby enhance the estimation of the concentration of chiral molecules in solution. Such a system can have potential for enabling low-cost, mobile chiral molecule concentration analysis, which would be wellsuited for a wide range of industrial and clinical applications where field testing or on-site testing is required.
\end{abstract}

\section{Introduction}

Polarimetry is a common tool for determining the concentration of chiral molecules; for example, organic molecules such as amino acids, lipids, sugars, and cholesterol. As such, polarimetry systems have been an important tool in a wide range of applications, such as flavor testing in the food industry, urine analysis in clinics and hospitals, sugar purity testing in sugar refineries, and as teaching tools in universities and colleges. One of the main barriers to the widespread use of polarimetry systems for chiral molecule concentration estimation for applications where mobility is key, such as field testing or on-site testing, is that a majority of current systems are large, complex, and costly, thus limiting their field portability. The main contributors to the size and complexity of such system are the multitude of mechanical parts used to enhance the measurements, and the main contributor to the cost is the use of complex optical components to isolate the polarisation state. While field-portable polarimetry systems do exist, they are manual systems that are subject to operator error and requires further calculations to determine the chiral molecule concentrations. As such, field-portable polarimetry systems that can automatically provide reliable estimates of chiral molecules are highly desired.

While computational models do exist to remove operator bias and enhance polarimetric measurements for concentration estimation, but they are rudimentary and do not leverage the spatial distribution of the laser intensity profile.

Motivated to achieve field-portable polarimetry for automatic chiral concentration assessment, we are motivated to leverage the tremendous advances in smartphone technology, which has seen incredible increases in not only computing power, but also in the resolution and sensitivity of the form-factor camera systems integrated into smartphones. This has made smartphones great platforms for developing smaller, field-portable devices that would have previously required separate large desktop/laptop computer systems and large camera systems. For example, there has been efforts in recent years to develop smartphone spectrometers and microscopes that scientists and industrial professionals can bring on-site to perform field studies. These small form factor devices not only make use of the smaller cameras embedded in smartphones, but also the computing power of smartphones to leverage computational methods for enhancing measurements, reducing uncertainties in state estimations, and inferring additional information beyond the captured data.

In this paper, we present a compact, field-portable smartphone chiral molecule concentration estimation system based on the principle of multi-sensor computational polarimetry. The proposed system contains a minimum set of static optical elements, leverages

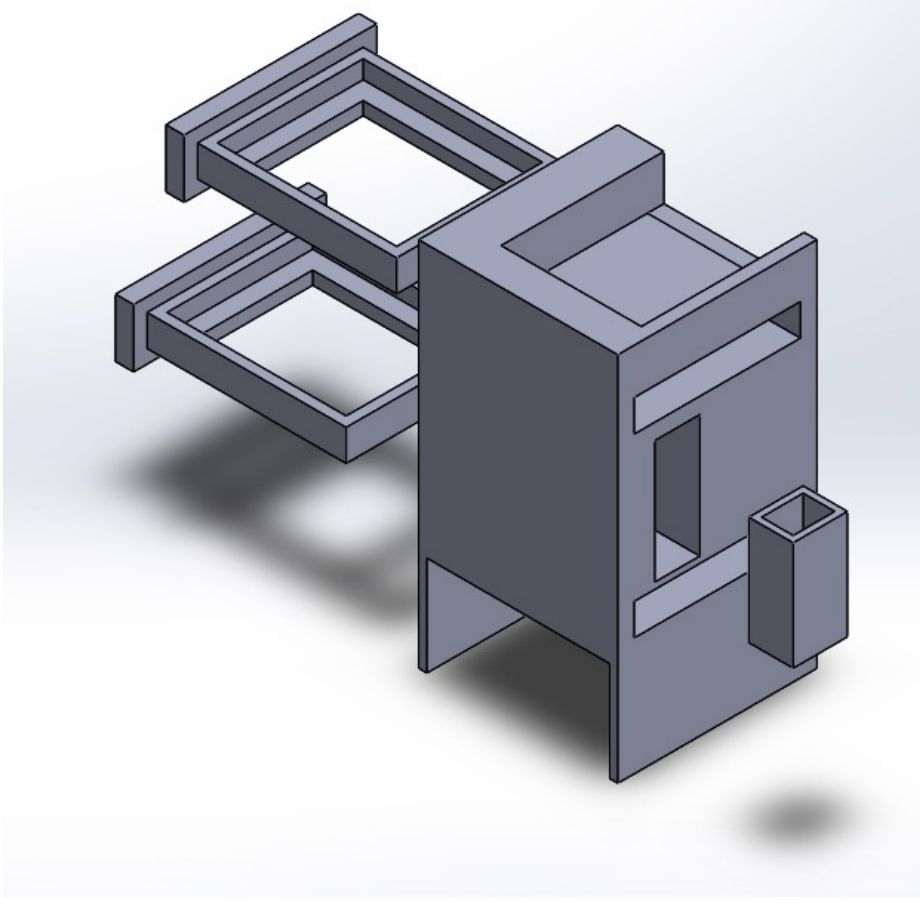

Fig. 1: Rendering of compact, field-portable smartphone chiral molecule concentration estimation system.

the smartphone camera to make high-resolution, multi-sensor acquisitions, and takes advantage of the computing power of smartphones to perform computational methods that leverage the spatial distribution of the laser intensity profile as captured by multisensor acquisitions to enhance measurements and estimate chiral molecule concentrations in a solution.

\section{Methodology}

In this section, the design of the presented compact, field-portable smartphone chiral molecule concentration estimation system, along with the computational method for estimating the angle of linear polarisation based on multi-sensor measurements as well as obtaining the subsequent chiral molecule concentration are described below.

\subsection{Chiral Molecule Concentration Estimation via Po- larimetry}

Chiral molecules are optically active, that is to say that they rotate the plane polarisation of light. The amount by which chiral molecules rotate light is proportional to the concentration of the chiral molecules and the distance the polarised light has travelled through the solution, and this relationship can be expressed using Boit's Law as

$$
\alpha=[\alpha]_{\lambda}^{T} c l
$$

where $[\alpha]_{\lambda}^{T}$ is the specific rotation of the chiral molecule at a particular temperature and wavelength, $c$ is the concentration of the chiral molecule in solution, and $l$ is the length of solution the polarised light has to travel through.

As mentioned earlier, manual polarimetry devices have been used to determine the concentration of the chiral molecules in solution. One such device is a disc polarimeter, which consists of 


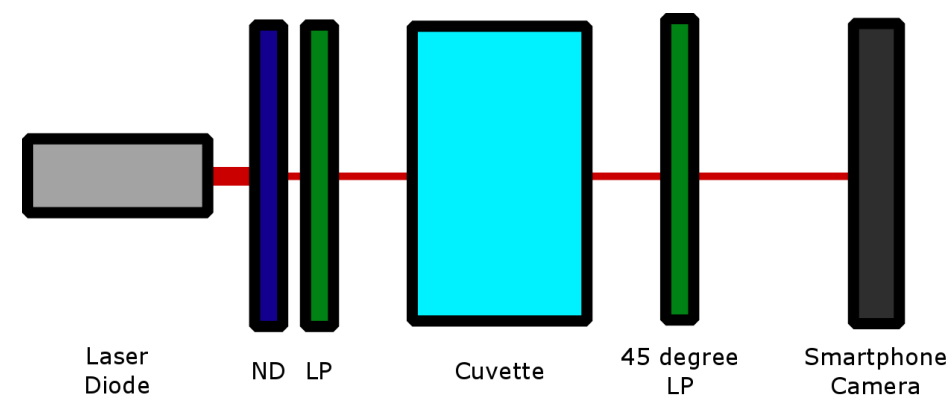

Fig. 2: Schematic of the optical setup. The system consists of: i) a diode laser source, ii) a linear polariser, iii) a cell for chiral solution, iv) a linear Analyser (45 degree offset from polariser), and a smartphone camera to obtain the multitude of sensor acquisitions.

aa light source, a static polariser, a rotating linear polariser (referred to as the analyser), and a sample tube filled with solution that is placed in between the polariser and the analyser. When the light source is turned on, the operator rotates the analyser until a uniform intensity pattern is revealed through the eyepiece. This amount of rotation is the amount that the chiral molecule solution has rotated the plane polarised light from the light source, with which one can then infer the chiral molecule concentration. Since the amount of rotation is read off of a vernier scale in such a device, such devices are easily susceptible to operator error with a sensitivity of $0.05^{\circ}$ typically.

More sophisticated chiral molecule concentration estimation systems have been developed that utilizes this same principle, where the rotation of the linear polariser is digitally controlled, and highly sensitive photodiodes are used to determine when the correct amount of rotation has been achieved by the polariser. These devices are fairly large, table-top based, and expensive; limiting their portability.

In the presented system, we take advantage of Maul's Law to reduce the amount of moving parts required, thus enabling a more compact, robust design. Maul's Law states that the intensity of a polarised beam $\left(I_{o}\right)$ through a rotated polariser is scaled proportionally to the square of the cosine of the amount of rotation $(\alpha)$ :

$$
I=I_{o} \cos ^{2} \alpha
$$

As such, we can use the inversion of this law to determine the angle of rotation caused by the chiral molecule solution,

$$
\alpha=\cos ^{-1} \sqrt{\frac{I}{I_{o}}}
$$

\subsection{System Design}

The presented compact, field-portable smartphone chiral molecule concentration estimation system is based on multi-sensor computational polarimetry, where a multitude of sensor acquisitions at different spatial locations are used to enhance measurements such that more reliable chiral molecule concentration estimates can be obtained. A rendering of the presented system, which acts as an attachment to a smartphone and holds all of the static optical elements and smartphone in a fixed configuration, is shown in Figure 1. The optical setup for the presented system is shown in Figure 2 .

The system consists of four static components in addition to the smartphone: i) a diode laser source (in this particular design, a $635 \mathrm{~nm}$ CPS635 laser from Thorlabs, NJ), ii) a linear polariser, iii) a cell for chiral solution, and iv) a linear analyser (configured at a $45^{\circ}$ offset from the polariser). While a typical chiral concentration estimation system makes use of a rotating polariser, we instead chose a static polariser for the presented system to keep the system as compact as possible as well as to reduce the number of overall acquisitions required.

The presented device is set up such as the laser diode comes from underneath the system and have the smartphone is placed on the top of the system to allow for ease-of-use. Once the smartphone camera makes the multitude of sensor measurements via its high-resolution two-dimensional detector array, the computational method proposed by Haider et al. [1] is used to provide an estimate of the chiral molecule concentration based on the multitude of sensor acquisitions.

\subsection{Enhanced measurements via Multi-sensor Compu- tational Polarimetry}

In the large tabletop devices for chiral molecule concentration estimation, time averaging is typically used to reduce detector and flicker noise that can cause stochastic fluctuations in the intensity readings from the photodiode. This is especially important due to the high sensitivity of the photodiode used in such systems. However, such methods require a multitude of acquisitions over time. In the presented system, rather than making use of time averaging to enhance measurements and thus obtain more reliable concentration estimates, we instead take advantage of the fact that smartphones are equipped with embedded camera systems that have high-resolution two-dimensional detector arrays and leverage the computational method proposed by Haider et al. [1], which utilizes the multi-sensor measurements made using only a single acquisition along with a priori knowledge with regards to the spatial distribution of the laser intensity profile to enhance estimates of the angle of linear polarisation.

\section{Device Considerations}

When taking advantage of consumer electronics and devices for scientific applications, the user has to be wary of inherent limitations of these devices since they are being used outside of their original use cases. This section will outline two such considerations in the development of a smartphone chiral molecule concentration estimation system.

\subsection{Bit Depth}

When a detector is converting photons into electrical signals, the digital sensitivity is described by the analog-to-digital converter (ADC) on the readout electronics. The amount of bits that this register can read out dictates the limit on the sensitivity of the camera to changes in intensity.

In relation to the presented system, the angular resolution of the device is limited by this ADC as well. An experiment can quickly show this limitation: imagine two ADCs from the same detector are limited to 8-bits and 16-bits, respectively, and the initial intensity of the polarised beam saturates the detector to the highest readout possible on either detector (i.e. $2^{8}-1$ and $2^{1} 6-1$, respectively), the question is how many degrees does the plane polarisation need to be rotated in order for the intensity to decrease by one intensity unit on the ADC. The amount of change for the 8-bit ADC is $3.56^{\circ}$ and for the 16 -bit $A D C$ is $0.22^{\circ}$

Depending on the chiral molecule and the application, an 8bit ADC is appropriate for the task, while in others, like in bloodglucose estimation or sugar purity analysis, a 16-bit ADC is more appropriate. In the current realization of the presented system, a Hauwei Nexus 6P smartphone was used, which has an ADC of 16-bit and images can be extracted in their native digital negative formats to maintain that bit-depth.

\subsection{Bayer Color Filter Array}

Almost all smartphone camera systems have detectors that have color filter arrays based on the Bayer pattern, where RGB color filters are arranged on top of the detector's photosites to separate light into the familiar red, green, and blue colours, thus allowing colour images to be produced. Cameras use digital shutters to read off information which allow for shutter speeds in the tenths of microseconds from the detector; however, in some resource-limited scenarios, the laser diode used in the presented system (which has a spectral wavelength of $635 \mathrm{~nm}$ and thus is within the red range) can still be too powerful and saturate the photosites with high spectral sensitivity to that particular wavelength due to the associated colour filter (e.g., red filter) in the Bayer color filter array. Fortunately, the filters used in the Bayer colour filter array have spectral sensitivity characteristics such as the red, green, and blue filters have overlapping regions in the spectrum, and thus light from the laser diode will not saturate photosites with low spectral sensitivity to that particular wavelength due to the associated colour filter (e.g., green and blue filters) in the Bayer color filter array, which in this case act as additional neutral density filters. 


\section{Conclusion}

In this paper, we presented a compact, field-portable, multi-sensor computational polarimetry system for estimating the concentration of chiral molecules that takes advantage of the powerful computing capabilities of a smartphone and the high-resolution imaging capabilities of a smartphone camera, as well as leverages Maul's Law to eliminate any dynamic parts, thereby decreasing the cost and complexity of the system. Design considerations and device considerations were discussed with regards to leveraging consumer devices such as smartphones as part of the presented system. Such a system can have potential for enabling low-cost, mobile chiral molecule concentration analysis, which would be well-suited for a wide range of industrial and clinical applications where field testing or on-site testing is required.

\section{Acknowledgments}

This work was supported by the Natural Sciences and Engineering Research Council of Canada, Canada Research Chairs Program, and the Ontario Ministry of Research and Innovation. The authors would like to thank their industry partner Lumalytics Inc.

\section{References}

[1] Haider, S.A., and Kazemzadeh, F., Clausi, D.A., and Wong, A. Integrated Computational Imaging System For Enhanced Polarimetric Measurements. SPIE Optical Engineering + Applications (2015). 